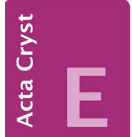
COMMUNICATIONS

ISSN 2056-9890

\section{Crystal structure of $(E)$-5-diethylamino- 2-(\{[4-(dimethylamino)phenyl]imino\}- methyl)phenol}

\author{
C. Vidya Rani, ${ }^{a}$ G. Chakkaravarthi ${ }^{\mathrm{b} *}$ and G. Rajagopal ${ }^{\mathrm{a} *}$ \\ aPG \& Research Department of Chemistry, Chikkanna Government Arts College, \\ Tiruppur 641 602, India, and 'bepartment of Physics, CPCL Polytechnic College, \\ Chennai 600 068, India. *Correspondence e-mail: \\ chakkaravarthi_2005@yahoo.com,rajagopal18@yahoo.com \\ Received 15 June 2015; accepted 19 June 2015
}

Edited by H. Stoeckli-Evans, University of Neuchâtel, Switzerland

The title Schiff base compound, $\mathrm{C}_{19} \mathrm{H}_{25} \mathrm{~N}_{3} \mathrm{O}$, is approximately planar, with a dihedral angle of $9.03(13)^{\circ}$ between the planes of the aromatic rings, and has an $E$ conformation about the $\mathrm{N}=\mathrm{C}$ bond. The molecular structure is stabilized by an intramolecular $\mathrm{O}-\mathrm{H} \cdots \mathrm{N}$ hydrogen bond, with an $S(6)$ ring motif. In the crystal, molecules are linked by $\mathrm{C}-\mathrm{H} \cdots \pi$ interactions, forming sheets parallel to the $b c$ plane.

Keywords: crystal structure; Schiff base; intramolecular $\mathrm{O}-\mathrm{H} \cdots \mathrm{N}$ hydrogen bond; $\mathrm{C}-\mathrm{H} \cdots \pi$ interactions.

CCDC reference: 1407678

\section{Related literature}

For biological activities of Schiff base derivatives, see: Savaliya et al. (2010); $\mathrm{Xu}$ et al. (2012). For the structures of similar compounds, see: Manvizhi et al. (2011); Thirugnanasundar et al. (2011).

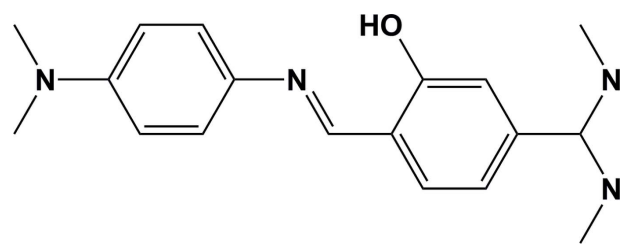

\section{Experimental}

\subsection{Crystal data}

$\mathrm{C}_{19} \mathrm{H}_{25} \mathrm{~N}_{3} \mathrm{O}$

$M_{r}=311.42$

Monoclinic, $P 2_{1}$

$a=8.8201$ (7) $\AA$

$$
\begin{aligned}
& b=7.8850(7) \AA \\
& c=13.0639(10) \AA \\
& \beta=108.407(3)^{\circ} \\
& V=862.06(12) \AA^{3}
\end{aligned}
$$

$Z=2$

Mo $K \alpha$ radiation

$\mu=0.08 \mathrm{~mm}^{-1}$

\subsection{Data collection}

Bruker Kappa APEXII CCD diffractometer

Absorption correction: multi-scan (SADABS; Sheldrick, 1996)

$T_{\min }=0.981, T_{\max }=0.985$

\subsection{Refinement}

$R\left[F^{2}>2 \sigma\left(F^{2}\right)\right]=0.052$

$w R\left(F^{2}\right)=0.159$

$S=1.03$

3825 reflections

214 parameters

$T=295 \mathrm{~K}$

$0.26 \times 0.22 \times 0.20 \mathrm{~mm}$

13009 measured reflections 3825 independent reflections 2438 reflections with $I>2 \sigma(I)$ $R_{\text {int }}=0.027$

Table 1

Hydrogen-bond geometry $\left(\AA,^{\circ}\right)$.

$C g 1$ and $C g 2$ are the centroids of rings $\mathrm{C} 3-\mathrm{C} 8$ and $\mathrm{C} 10-\mathrm{C} 15$, respectively.

\begin{tabular}{lllll}
\hline$D-\mathrm{H} \cdots A$ & $D-\mathrm{H}$ & $\mathrm{H} \cdots A$ & $D \cdots A$ & $D-\mathrm{H} \cdots A$ \\
\hline $\mathrm{O} 1-\mathrm{H} 1 \cdots \mathrm{N} 2$ & 0.82 & 1.85 & $2.585(3)$ & 148 \\
$\mathrm{C} 11-\mathrm{H} 11 \cdots C g 1^{\mathrm{i}}$ & 0.93 & 2.71 & $3.517(3)$ & 145 \\
$\mathrm{C} 17-\mathrm{H} 17 B \cdots C g 2^{\mathrm{ii}}$ & 0.96 & 2.90 & $3.743(5)$ & 147 \\
\hline
\end{tabular}

Symmetry codes: (i) $-x, y+\frac{1}{2},-z+1$; (ii) $-x, y-\frac{1}{2},-z+2$.

Data collection: APEX2 (Bruker, 2004); cell refinement: SAINT (Bruker, 2004); data reduction: $S A I N T$; $\operatorname{program}(\mathrm{s})$ used to solve structure: SHELXS97 (Sheldrick, 2008); program(s) used to refine structure: SHELXL97 (Sheldrick, 2008); molecular graphics: PLATON (Spek, 2009) and Mercury (Macrae et al., 2008); software used to prepare material for publication: SHELXL97 and PLATON.

\section{Acknowledgements}

The authors acknowledge the SAIF, IIT, Madras, for the data collection.

Supporting information for this paper is available from the IUCr electronic archives (Reference: SU5157).

\section{References}

Bruker (2004). APEX2 and SAINT. Bruker AXS Inc., Madison, Wisconsin, USA.

Macrae, C. F., Bruno, I. J., Chisholm, J. A., Edgington, P. R., McCabe, P., Pidcock, E., Rodriguez-Monge, L., Taylor, R., van de Streek, J. \& Wood, P. A. (2008). J. Appl. Cryst. 41, 466-470.

Manvizhi, K., Chakkaravarthi, G., Anbalagan, G. \& Rajagopal, G. (2011). Acta Cryst. E67, o2500.

Savaliya, M. D., Dobaria, J. G. \& Purohit, D. M. (2010). An Indian J. 6, 267271.

Sheldrick, G. M. (1996). SADABS. University of Göttingen, Germany.

Sheldrick, G. M. (2008). Acta Cryst. A64, 112-122.

Spek, A. L. (2009). Acta Cryst. D65, 148-155.

Thirugnanasundar, A., Suresh, J., Ramu, A. \& RajaGopal, G. (2011). Acta Cryst. E67, o2303.

Xu, R.-B., Zhang, N., Zhou, H.-Y., Yang, S.-P., Li, Y.-Y., Shi, D.-H., Ma, W.-X. \& Xu, X.-Y. (2012). J. Chem. Crystallogr. 42, 928-932. 


\section{supporting information}

Acta Cryst. (2015). E71, o503 [doi:10.1107/S2056989015011779]

\section{Crystal structure of $(E)-5$-diethylamino-2-(\{[4-(dimethylamino)phenyl]imino\}- methyl)phenol}

\section{Vidya Rani, G. Chakkaravarthi and G. Rajagopal}

\section{S1. Structural commentary}

Schiff base derivatives are known to exhibit antimicrobial (Savaliya et al., 2010) and antibacterial (Xu et al., 2012) activities. Herein we report on the synthesis and the crystal structure of a new Schiff base compound.

The molecular structure of the title compound is illustrated in Fg. 1. The geometric parameters are comparable to those reported for similar structures (Manvizhi et al., 2011; Thirugnanasundar et al., 2011). The dihedral angle between the benzene rings $(\mathrm{C} 3-\mathrm{C} 8)$ and $(\mathrm{C} 10-\mathrm{C} 15)$ is $9.03(13)^{\circ}$. The molecular structure is stabilized by an intramolecular $\mathrm{O}-$ $\mathrm{H} \cdots \mathrm{N}$ hydrogen bond (Table 1 and Fig. 1).

In the crystal, molecules are linked by $\mathrm{C}-\mathrm{H} \cdots \pi$ interactions forming sheets parallel to the bc plane (Table 1 and Fig. 2).

\section{S2. Synthesis and crystallization}

To an ethanol solution $(10 \mathrm{ml})$ of 5-(diethylamino)-2-hydroxybenzaldehyde $(96.5 \mathrm{mg}, 0.5 \mathrm{~mol})$ was added $N^{I}, N^{I}$-dimethylbenzene-1,4-diamine ( $68 \mathrm{mg}, 0.5 \mathrm{~mol}$ ). The mixture was stirred and 2 to 3 drops of glacial acetic acid were added. Stirring was continued for 30 mins and then the reaction mixture was refluxed for $2 \mathrm{~h}$. On completion of the reaction, monitored by TLC, the mixture was allowed to cool to room temperature and the solid yellow precipitate that formed was filtered, dried, and recrystallized from DMF, giving colourless block-like crystals.

\section{S3. Refinement}

Crystal data, data collection and structure refinement details are summarized in Table 2. $\mathrm{H}$ atoms were positioned geometrically and refined using riding model: $\mathrm{O}-\mathrm{H}=0.82 \AA, \mathrm{C}-\mathrm{H}=0.93-0.97 \AA$ with $U_{\text {iso }}(\mathrm{H})=1.5 \mathrm{Ueq}(\mathrm{O}, \mathrm{C})$ for the hydroxyl and methyl $\mathrm{H}$ atoms and $1.2 \mathrm{U}_{\mathrm{eq}}(\mathrm{C})$ for other $\mathrm{H}$ atoms. The components of the anisotropic displacement parameters of the atoms in bonds $\mathrm{N} 3-\mathrm{C} 16, \mathrm{~N} 3-\mathrm{C} 18$ and $\mathrm{N} 1-\mathrm{C} 2$ were restrained to be equal within an effective standard deviation of 0.001 using the DELU command, and the $\mathrm{C} 16-\mathrm{C} 17$ bond distance was restrained to 1.54 (1) $\AA$. 


\section{supporting information}

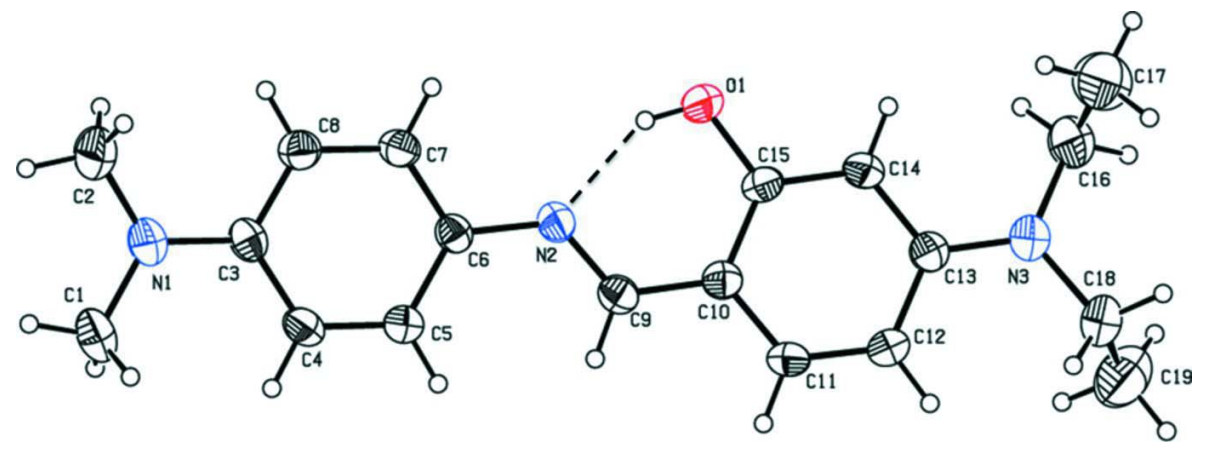

\section{Figure 1}

The molecular structure of the title compound, with atom labelling. Displacement ellipsoids are drawn at the 30\% probability level. The intramolecular $\mathrm{O}-\mathrm{H} . . \mathrm{N}$ hydrogen bonds is shown as a dashd lines (see Table 1 for details). 


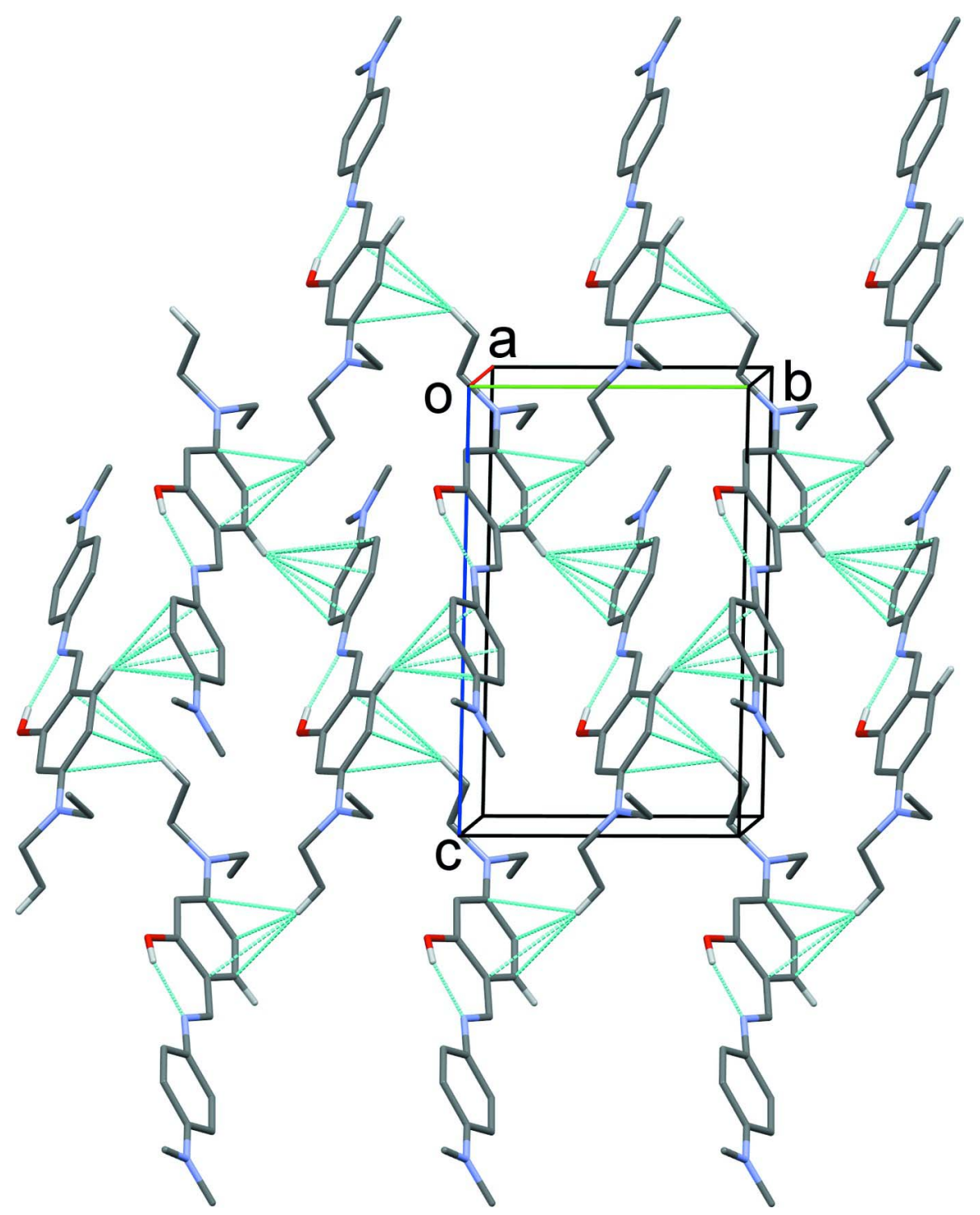

Figure 2

A view along the $a$ axis of the crystal apcking of the title compound. The $\mathrm{O}-\mathrm{H} . . \mathrm{N}$ and $\mathrm{C}-\mathrm{H} \cdots \pi$ interactions are illustrated by dashed lines (see Table 1 for details).

\section{(E)-5-Diethylamino-2-(\{[4-(dimethylamino)phenyl]imino\}methyl)phenol}

Crystal data

$\mathrm{C}_{19} \mathrm{H}_{25} \mathrm{~N}_{3} \mathrm{O}$

$M_{r}=311.42$

Monoclinic, $P 2_{1}$

Hall symbol: $\mathrm{P} 2 \mathrm{yb}$

$a=8.8201$ (7) $\AA$

$b=7.8850$ (7) $\AA$

$c=13.0639(10) \AA$

$\beta=108.407(3)^{\circ}$

$V=862.06(12) \AA^{3}$

$Z=2$
$F(000)=336$

$D_{\mathrm{x}}=1.200 \mathrm{Mg} \mathrm{m}^{-3}$

Mo $K \alpha$ radiation, $\lambda=0.71073 \AA$

Cell parameters from 4428 reflections

$\theta=2.4-27.2^{\circ}$

$\mu=0.08 \mathrm{~mm}^{-1}$

$T=295 \mathrm{~K}$

Block, colourless

$0.26 \times 0.22 \times 0.20 \mathrm{~mm}$ 


\section{Data collection}

Bruker Kappa APEXII CCD diffractometer

Radiation source: fine-focus sealed tube Graphite monochromator

$\omega$ and $\varphi$ scan

Absorption correction: multi-scan

(SADABS; Sheldrick, 1996)

$T_{\min }=0.981, T_{\max }=0.985$

\section{Refinement}

Refinement on $F^{2}$

Least-squares matrix: full

$R\left[F^{2}>2 \sigma\left(F^{2}\right)\right]=0.052$

$w R\left(F^{2}\right)=0.159$

$S=1.03$

3825 reflections

214 parameters

6 restraints

Primary atom site location: structure-invariant direct methods

Secondary atom site location: difference Fourier map

\section{Special details}

Geometry. All esds (except the esd in the dihedral angle between two 1.s. planes) are estimated using the full covariance matrix. The cell esds are taken into account individually in the estimation of esds in distances, angles and torsion angles; correlations between esds in cell parameters are only used when they are defined by crystal symmetry. An approximate (isotropic) treatment of cell esds is used for estimating esds involving l.s. planes.

Refinement. Refinement of $\mathrm{F}^{2}$ against ALL reflections. The weighted R-factor $\mathrm{wR}$ and goodness of fit $\mathrm{S}$ are based on $\mathrm{F}^{2}$, conventional R-factors $R$ are based on $F$, with $F$ set to zero for negative $F^{2}$. The threshold expression of $F^{2}>2 \operatorname{sigma}\left(F^{2}\right)$ is used only for calculating R-factors ( $\mathrm{gt}$ ) etc. and is not relevant to the choice of reflections for refinement. R-factors based on $\mathrm{F}^{2}$ are statistically about twice as large as those based on F, and R- factors based on ALL data will be even larger.

Fractional atomic coordinates and isotropic or equivalent isotropic displacement parameters $\left(\AA^{2}\right)$

\begin{tabular}{lllll}
\hline & $x$ & $y$ & $z$ & $U_{\text {iso }} * / U_{\text {eq }}$ \\
\hline C1 & $0.4431(4)$ & $0.6814(5)$ & $0.2015(2)$ & $0.0780(10)$ \\
H1A & 0.3484 & 0.6253 & 0.1573 & $0.117^{*}$ \\
H1B & 0.5213 & 0.6840 & 0.1644 & $0.117^{*}$ \\
H1C & 0.4171 & 0.7953 & 0.2159 & $0.117^{*}$ \\
C2 & $0.6641(4)$ & $0.5264(6)$ & $0.3287(3)$ & $0.0902(12)$ \\
H2A & 0.7316 & 0.5867 & 0.3901 & $0.135^{*}$ \\
H2B & 0.7039 & 0.5405 & 0.2688 & $0.135^{*}$ \\
H2C & 0.6635 & 0.4081 & 0.3459 & $0.135^{*}$ \\
C3 & $0.4220(3)$ & $0.5850(3)$ & $0.3736(2)$ & $0.0502(6)$ \\
C4 & $0.2713(3)$ & $0.6598(4)$ & $0.3525(2)$ & $0.0547(7)$ \\
H4 & 0.2275 & 0.7180 & 0.2880 & $0.066^{*}$ \\
C5 & $0.1861(3)$ & $0.6502(4)$ & $0.42371(19)$ & $0.0512(6)$ \\
H5 & 0.0860 & 0.7008 & 0.4067 & $0.061^{*}$ \\
C6 & $0.2487(3)$ & $0.5648(3)$ & $0.52175(18)$ & $0.0444(6)$ \\
C7 & $0.3965(3)$ & $0.4922(3)$ & $0.5425(2)$ & $0.0508(6)$
\end{tabular}

13009 measured reflections

$R_{\text {int }}=0.027$

$\theta_{\max }=27.3^{\circ}, \theta_{\min }=2.4^{\circ}$

$h=-11 \rightarrow 10$

$=-10 \rightarrow 10$

Hydrogen site location: inferred from neighbouring sites

$\mathrm{H}$-atom parameters constrained

$w=1 /\left[\sigma^{2}\left(F_{\mathrm{o}}^{2}\right)+(0.0751 P)^{2}+0.1538 P\right]$

where $P=\left(F_{\mathrm{o}}^{2}+2 F_{\mathrm{c}}^{2}\right) / 3$

每 $<0.001$

$\Delta \rho_{\min }=-0.16$ e $\AA^{-3}$

Extinction correction: SHELXL97 (Sheldrick, 2008), $\mathrm{Fc}^{*}=\mathrm{kFc}\left[1+0.001 \times \mathrm{Fc}^{2} \lambda^{3} / \sin (2 \theta)\right]^{-1 / 4}$

Extinction coefficient: 0.014 (4)
3825 independent reflections 


$\begin{array}{lllll}\text { H7 } & 0.4405 & 0.4352 & 0.6075 & 0.061^{*} \\ \text { C8 } & 0.4820(3) & 0.5003(4) & 0.4715(2) & 0.0537(7) \\ \text { H8 } & 0.5816 & 0.4483 & 0.4889 & 0.064^{*} \\ \text { C9 } & 0.0435(3) & 0.6249(4) & 0.59847(19) & 0.0481(6) \\ \text { H9 } & -0.0034 & 0.6975 & 0.5411 & 0.058^{*} \\ \text { C10 } & -0.0294(3) & 0.6066(3) & 0.68154(19) & 0.0462(6) \\ \text { C11 } & -0.1669(3) & 0.6947(3) & 0.6784(2) & 0.0537(7) \\ \text { H11 } & -0.2150 & 0.7626 & 0.6188 & 0.064^{*} \\ \text { C12 } & -0.2347(3) & 0.6864(4) & 0.7586(2) & 0.0596(8) \\ \text { H12 } & -0.3278 & 0.7467 & 0.7524 & 0.072^{*} \\ \text { C13 } & -0.1648(3) & 0.5871(4) & 0.8507(2) & 0.0528(6) \\ \text { C14 } & -0.0279(3) & 0.4940(3) & 0.8547(2) & 0.0523(7) \\ \text { H14 } & 0.0198 & 0.4255 & 0.9141 & 0.063^{*} \\ \text { C15 } & 0.0370(3) & 0.5025(3) & 0.7722(2) & 0.0472(6) \\ \text { C16 } & -0.1784(4) & 0.4511(5) & 1.0194(3) & 0.0834(10) \\ \text { H16A } & -0.2702 & 0.4176 & 1.0403 & 0.100^{*} \\ \text { H16B } & -0.1393 & 0.3520 & 0.9915 & 0.100^{*} \\ \text { C17 } & -0.0541(5) & 0.5166(6) & 1.1125(3) & 0.1089(14) \\ \text { H17A } & 0.0389 & 0.5432 & 1.0925 & 0.163^{*} \\ \text { H17B } & -0.0274 & 0.4327 & 1.1686 & 0.163^{*} \\ \text { H17C } & -0.0917 & 0.6173 & 1.1380 & 0.163^{*} \\ \text { C18 } & -0.3507(4) & 0.7080(5) & 0.9409(3) & 0.0835(10) \\ \text { H18A } & -0.3375 & 0.7326 & 1.0160 & 0.100^{*} \\ \text { H18B } & -0.3372 & 0.8131 & 0.9062 & 0.100^{*} \\ \text { C19 } & -0.5106(4) & 0.6420(8) & 0.8890(4) & 0.1178(15) \\ \text { H19A } & -0.5223 & 0.6129 & 0.8156 & 0.177^{*} \\ \text { H19B } & -0.5881 & 0.7267 & 0.8905 & 0.177^{*} \\ \text { H19C } & -0.5270 & 0.5428 & 0.9268 & 0.177^{*} \\ \text { N1 } & 0.5063(3) & 0.5915(4) & 0.30117(18) & 0.0693(7) \\ \text { N2 } & 0.1707(2) & 0.5451(3) & 0.60028(16) & 0.0495(5) \\ \text { N3 } & -0.2261(3) & 0.5836(4) & 0.9344(2) & 0.0826(9) \\ \text { O1 } & 0.1678(2) & 0.4075(3) & 0.77919(17) & 0.0695(6) \\ \text { H1 } & 0.2010 & 0.4308 & 0.7289 & 0.104^{*} \\ & & & & \end{array}$

Atomic displacement parameters $\left(\AA^{2}\right)$

\begin{tabular}{lllllll}
\hline & $U^{11}$ & $U^{22}$ & $U^{33}$ & $U^{12}$ & $U^{13}$ & $U^{23}$ \\
\hline C1 & $0.097(2)$ & $0.090(3)$ & $0.0568(17)$ & $-0.0042(19)$ & $0.0384(16)$ & $0.0063(17)$ \\
C2 & $0.0743(17)$ & $0.127(3)$ & $0.082(2)$ & $0.0100(19)$ & $0.0428(18)$ & $0.012(2)$ \\
C3 & $0.0561(14)$ & $0.0511(15)$ & $0.0454(13)$ & $-0.0092(13)$ & $0.0189(11)$ & $0.0004(12)$ \\
C4 & $0.0608(16)$ & $0.0587(17)$ & $0.0431(13)$ & $0.0034(14)$ & $0.0143(12)$ & $0.0113(13)$ \\
C5 & $0.0487(13)$ & $0.0557(16)$ & $0.0487(14)$ & $0.0064(12)$ & $0.0148(11)$ & $0.0060(13)$ \\
C6 & $0.0510(14)$ & $0.0410(14)$ & $0.0418(13)$ & $-0.0028(12)$ & $0.0154(11)$ & $0.0013(11)$ \\
C7 & $0.0506(14)$ & $0.0535(16)$ & $0.0453(14)$ & $0.0033(13)$ & $0.0110(11)$ & $0.0073(12)$ \\
C8 & $0.0494(14)$ & $0.0554(16)$ & $0.0544(16)$ & $0.0018(12)$ & $0.0137(12)$ & $0.0038(13)$ \\
C9 & $0.0505(14)$ & $0.0473(15)$ & $0.0436(13)$ & $-0.0018(13)$ & $0.0107(11)$ & $0.0001(11)$ \\
C10 & $0.0455(13)$ & $0.0444(14)$ & $0.0467(13)$ & $-0.0009(11)$ & $0.0116(10)$ & $-0.0012(11)$ \\
C11 & $0.0547(14)$ & $0.0533(16)$ & $0.0496(14)$ & $0.0138(12)$ & $0.0113(12)$ & $0.0110(12)$
\end{tabular}


supporting information

\begin{tabular}{lllllll}
$\mathrm{C} 12$ & $0.0529(14)$ & $0.066(2)$ & $0.0604(16)$ & $0.0188(13)$ & $0.0188(13)$ & $0.0095(14)$ \\
$\mathrm{C} 13$ & $0.0533(14)$ & $0.0548(16)$ & $0.0530(14)$ & $0.0039(13)$ & $0.0207(11)$ & $0.0075(13)$ \\
$\mathrm{C} 14$ & $0.0543(15)$ & $0.0523(16)$ & $0.0506(15)$ & $0.0101(13)$ & $0.0167(12)$ & $0.0147(13)$ \\
$\mathrm{C} 15$ & $0.0403(12)$ & $0.0443(14)$ & $0.0554(15)$ & $0.0053(11)$ & $0.0129(11)$ & $0.0050(12)$ \\
C16 & $0.086(2)$ & $0.093(3)$ & $0.081(2)$ & $0.0042(18)$ & $0.0414(19)$ & $0.0168(16)$ \\
C17 & $0.126(3)$ & $0.098(3)$ & $0.097(3)$ & $0.010(3)$ & $0.028(3)$ & $-0.008(3)$ \\
C18 & $0.080(2)$ & $0.107(3)$ & $0.075(2)$ & $0.0190(17)$ & $0.0400(18)$ & $0.0039(19)$ \\
C19 & $0.094(3)$ & $0.134(4)$ & $0.131(4)$ & $0.014(3)$ & $0.043(3)$ & $-0.015(3)$ \\
N1 & $0.0737(14)$ & $0.083(2)$ & $0.0611(15)$ & $0.0044(13)$ & $0.0357(13)$ & $0.0146(14)$ \\
N2 & $0.0530(12)$ & $0.0515(14)$ & $0.0459(11)$ & $0.0007(10)$ & $0.0184(9)$ & $0.0030(10)$ \\
N3 & $0.0769(16)$ & $0.112(2)$ & $0.0696(16)$ & $0.0288(15)$ & $0.0381(13)$ & $0.0210(15)$ \\
O1 & $0.0666(12)$ & $0.0777(14)$ & $0.0737(14)$ & $0.0302(11)$ & $0.0356(10)$ & $0.0283(11)$ \\
\hline
\end{tabular}

Geometric parameters $\left(\AA,{ }^{\circ}\right)$

\begin{tabular}{|c|c|c|c|}
\hline $\mathrm{C} 1-\mathrm{N} 1$ & $1.432(4)$ & $\mathrm{C} 11-\mathrm{C} 12$ & $1.363(4)$ \\
\hline $\mathrm{C} 1-\mathrm{H} 1 \mathrm{~A}$ & 0.9600 & $\mathrm{C} 11-\mathrm{H} 11$ & 0.9300 \\
\hline $\mathrm{C} 1-\mathrm{H} 1 \mathrm{~B}$ & 0.9600 & $\mathrm{C} 12-\mathrm{C} 13$ & $1.404(4)$ \\
\hline $\mathrm{C} 1-\mathrm{H} 1 \mathrm{C}$ & 0.9600 & $\mathrm{C} 12-\mathrm{H} 12$ & 0.9300 \\
\hline $\mathrm{C} 2-\mathrm{N} 1$ & $1.419(4)$ & $\mathrm{C} 13-\mathrm{N} 3$ & $1.364(3)$ \\
\hline $\mathrm{C} 2-\mathrm{H} 2 \mathrm{~A}$ & 0.9600 & $\mathrm{C} 13-\mathrm{C} 14$ & $1.400(3)$ \\
\hline $\mathrm{C} 2-\mathrm{H} 2 \mathrm{~B}$ & 0.9600 & $\mathrm{C} 14-\mathrm{C} 15$ & $1.373(4)$ \\
\hline $\mathrm{C} 2-\mathrm{H} 2 \mathrm{C}$ & 0.9600 & C14-H14 & 0.9300 \\
\hline $\mathrm{C} 3-\mathrm{N} 1$ & $1.377(3)$ & $\mathrm{C} 15-\mathrm{O} 1$ & $1.354(3)$ \\
\hline $\mathrm{C} 3-\mathrm{C} 8$ & $1.390(4)$ & $\mathrm{C} 16-\mathrm{C} 17$ & $1.451(5)$ \\
\hline $\mathrm{C} 3-\mathrm{C} 4$ & $1.400(4)$ & $\mathrm{C} 16-\mathrm{N} 3$ & $1.485(4)$ \\
\hline $\mathrm{C} 4-\mathrm{C} 5$ & $1.370(4)$ & $\mathrm{C} 16-\mathrm{H} 16 \mathrm{~A}$ & 0.9700 \\
\hline $\mathrm{C} 4-\mathrm{H} 4$ & 0.9300 & $\mathrm{C} 16-\mathrm{H} 16 \mathrm{~B}$ & 0.9700 \\
\hline $\mathrm{C} 5-\mathrm{C} 6$ & $1.397(3)$ & C17-H17A & 0.9600 \\
\hline $\mathrm{C} 5-\mathrm{H} 5$ & 0.9300 & C17-H17B & 0.9600 \\
\hline $\mathrm{C} 6-\mathrm{C} 7$ & $1.370(3)$ & $\mathrm{C} 17-\mathrm{H} 17 \mathrm{C}$ & 0.9600 \\
\hline $\mathrm{C} 6-\mathrm{N} 2$ & $1.413(3)$ & C18-C19 & $1.454(5)$ \\
\hline $\mathrm{C} 7-\mathrm{C} 8$ & $1.370(4)$ & $\mathrm{C} 18-\mathrm{N} 3$ & $1.495(4)$ \\
\hline $\mathrm{C} 7-\mathrm{H} 7$ & 0.9300 & C18-H18A & 0.9700 \\
\hline $\mathrm{C} 8-\mathrm{H} 8$ & 0.9300 & $\mathrm{C} 18-\mathrm{H} 18 \mathrm{~B}$ & 0.9700 \\
\hline $\mathrm{C} 9-\mathrm{N} 2$ & $1.280(3)$ & C19-H19A & 0.9600 \\
\hline $\mathrm{C} 9-\mathrm{C} 10$ & $1.433(3)$ & C19-H19B & 0.9600 \\
\hline C9- $\mathrm{H} 9$ & 0.9300 & $\mathrm{C} 19-\mathrm{H} 19 \mathrm{C}$ & 0.9600 \\
\hline $\mathrm{C} 10-\mathrm{C} 11$ & $1.386(3)$ & $\mathrm{O} 1-\mathrm{H} 1$ & 0.8200 \\
\hline $\mathrm{C} 10-\mathrm{C} 15$ & $1.407(3)$ & & \\
\hline $\mathrm{N} 1-\mathrm{C} 1-\mathrm{H} 1 \mathrm{~A}$ & 109.5 & $\mathrm{~N} 3-\mathrm{C} 13-\mathrm{C} 14$ & $121.0(2)$ \\
\hline $\mathrm{N} 1-\mathrm{C} 1-\mathrm{H} 1 \mathrm{~B}$ & 109.5 & $\mathrm{~N} 3-\mathrm{C} 13-\mathrm{C} 12$ & $121.3(2)$ \\
\hline $\mathrm{H} 1 \mathrm{~A}-\mathrm{C} 1-\mathrm{H} 1 \mathrm{~B}$ & 109.5 & $\mathrm{C} 14-\mathrm{C} 13-\mathrm{C} 12$ & $117.7(2)$ \\
\hline $\mathrm{N} 1-\mathrm{C} 1-\mathrm{H} 1 \mathrm{C}$ & 109.5 & $\mathrm{C} 15-\mathrm{C} 14-\mathrm{C} 13$ & $120.9(2)$ \\
\hline $\mathrm{H} 1 \mathrm{~A}-\mathrm{C} 1-\mathrm{H} 1 \mathrm{C}$ & 109.5 & $\mathrm{C} 15-\mathrm{C} 14-\mathrm{H} 14$ & 119.5 \\
\hline $\mathrm{H} 1 \mathrm{~B}-\mathrm{C} 1-\mathrm{H} 1 \mathrm{C}$ & 109.5 & $\mathrm{C} 13-\mathrm{C} 14-\mathrm{H} 14$ & 119.5 \\
\hline $\mathrm{N} 1-\mathrm{C} 2-\mathrm{H} 2 \mathrm{~A}$ & 109.5 & $\mathrm{O} 1-\mathrm{C} 15-\mathrm{C} 14$ & $118.5(2)$ \\
\hline
\end{tabular}




\begin{tabular}{|c|c|c|c|}
\hline $\mathrm{N} 1-\mathrm{C} 2-\mathrm{H} 2 \mathrm{~B}$ & 109.5 & $\mathrm{O} 1-\mathrm{C} 15-\mathrm{C} 10$ & $119.9(2)$ \\
\hline $\mathrm{H} 2 \mathrm{~A}-\mathrm{C} 2-\mathrm{H} 2 \mathrm{~B}$ & 109.5 & $\mathrm{C} 14-\mathrm{C} 15-\mathrm{C} 10$ & $121.6(2)$ \\
\hline $\mathrm{N} 1-\mathrm{C} 2-\mathrm{H} 2 \mathrm{C}$ & 109.5 & $\mathrm{C} 17-\mathrm{C} 16-\mathrm{N} 3$ & $109.7(3)$ \\
\hline $\mathrm{H} 2 \mathrm{~A}-\mathrm{C} 2-\mathrm{H} 2 \mathrm{C}$ & 109.5 & $\mathrm{C} 17-\mathrm{C} 16-\mathrm{H} 16 \mathrm{~A}$ & 109.7 \\
\hline $\mathrm{H} 2 \mathrm{~B}-\mathrm{C} 2-\mathrm{H} 2 \mathrm{C}$ & 109.5 & $\mathrm{~N} 3-\mathrm{C} 16-\mathrm{H} 16 \mathrm{~A}$ & 109.7 \\
\hline $\mathrm{N} 1-\mathrm{C} 3-\mathrm{C} 8$ & $121.2(2)$ & $\mathrm{C} 17-\mathrm{C} 16-\mathrm{H} 16 \mathrm{~B}$ & 109.7 \\
\hline $\mathrm{N} 1-\mathrm{C} 3-\mathrm{C} 4$ & $122.3(2)$ & $\mathrm{N} 3-\mathrm{C} 16-\mathrm{H} 16 \mathrm{~B}$ & 109.7 \\
\hline $\mathrm{C} 8-\mathrm{C} 3-\mathrm{C} 4$ & $116.4(2)$ & $\mathrm{H} 16 \mathrm{~A}-\mathrm{C} 16-\mathrm{H} 16 \mathrm{~B}$ & 108.2 \\
\hline $\mathrm{C} 5-\mathrm{C} 4-\mathrm{C} 3$ & $122.2(2)$ & $\mathrm{C} 16-\mathrm{C} 17-\mathrm{H} 17 \mathrm{~A}$ & 109.5 \\
\hline $\mathrm{C} 5-\mathrm{C} 4-\mathrm{H} 4$ & 118.9 & $\mathrm{C} 16-\mathrm{C} 17-\mathrm{H} 17 \mathrm{~B}$ & 109.5 \\
\hline $\mathrm{C} 3-\mathrm{C} 4-\mathrm{H} 4$ & 118.9 & $\mathrm{H} 17 \mathrm{~A}-\mathrm{C} 17-\mathrm{H} 17 \mathrm{~B}$ & 109.5 \\
\hline $\mathrm{C} 4-\mathrm{C} 5-\mathrm{C} 6$ & $120.4(2)$ & $\mathrm{C} 16-\mathrm{C} 17-\mathrm{H} 17 \mathrm{C}$ & 109.5 \\
\hline $\mathrm{C} 4-\mathrm{C} 5-\mathrm{H} 5$ & 119.8 & $\mathrm{H} 17 \mathrm{~A}-\mathrm{C} 17-\mathrm{H} 17 \mathrm{C}$ & 109.5 \\
\hline $\mathrm{C} 6-\mathrm{C} 5-\mathrm{H} 5$ & 119.8 & $\mathrm{H} 17 \mathrm{~B}-\mathrm{C} 17-\mathrm{H} 17 \mathrm{C}$ & 109.5 \\
\hline $\mathrm{C} 7-\mathrm{C} 6-\mathrm{C} 5$ & $117.3(2)$ & $\mathrm{C} 19-\mathrm{C} 18-\mathrm{N} 3$ & $111.1(3)$ \\
\hline $\mathrm{C} 7-\mathrm{C} 6-\mathrm{N} 2$ & $117.5(2)$ & $\mathrm{C} 19-\mathrm{C} 18-\mathrm{H} 18 \mathrm{~A}$ & 109.4 \\
\hline $\mathrm{C} 5-\mathrm{C} 6-\mathrm{N} 2$ & $125.2(2)$ & N3-C18-H18A & 109.4 \\
\hline $\mathrm{C} 8-\mathrm{C} 7-\mathrm{C} 6$ & $122.6(2)$ & $\mathrm{C} 19-\mathrm{C} 18-\mathrm{H} 18 \mathrm{~B}$ & 109.4 \\
\hline $\mathrm{C} 8-\mathrm{C} 7-\mathrm{H} 7$ & 118.7 & $\mathrm{~N} 3-\mathrm{C} 18-\mathrm{H} 18 \mathrm{~B}$ & 109.4 \\
\hline $\mathrm{C} 6-\mathrm{C} 7-\mathrm{H} 7$ & 118.7 & $\mathrm{H} 18 \mathrm{~A}-\mathrm{C} 18-\mathrm{H} 18 \mathrm{~B}$ & 108.0 \\
\hline $\mathrm{C} 7-\mathrm{C} 8-\mathrm{C} 3$ & $121.0(2)$ & $\mathrm{C} 18-\mathrm{C} 19-\mathrm{H} 19 \mathrm{~A}$ & 109.5 \\
\hline $\mathrm{C} 7-\mathrm{C} 8-\mathrm{H} 8$ & 119.5 & $\mathrm{C} 18-\mathrm{C} 19-\mathrm{H} 19 \mathrm{~B}$ & 109.5 \\
\hline $\mathrm{C} 3-\mathrm{C} 8-\mathrm{H} 8$ & 119.5 & $\mathrm{H} 19 \mathrm{~A}-\mathrm{C} 19-\mathrm{H} 19 \mathrm{~B}$ & 109.5 \\
\hline $\mathrm{N} 2-\mathrm{C} 9-\mathrm{C} 10$ & $122.5(2)$ & $\mathrm{C} 18-\mathrm{C} 19-\mathrm{H} 19 \mathrm{C}$ & 109.5 \\
\hline $\mathrm{N} 2-\mathrm{C} 9-\mathrm{H} 9$ & 118.8 & $\mathrm{H} 19 \mathrm{~A}-\mathrm{C} 19-\mathrm{H} 19 \mathrm{C}$ & 109.5 \\
\hline $\mathrm{C} 10-\mathrm{C} 9-\mathrm{H} 9$ & 118.8 & $\mathrm{H} 19 \mathrm{~B}-\mathrm{C} 19-\mathrm{H} 19 \mathrm{C}$ & 109.5 \\
\hline $\mathrm{C} 11-\mathrm{C} 10-\mathrm{C} 15$ & $116.4(2)$ & $\mathrm{C} 3-\mathrm{N} 1-\mathrm{C} 2$ & $120.8(2)$ \\
\hline $\mathrm{C} 11-\mathrm{C} 10-\mathrm{C} 9$ & $121.6(2)$ & $\mathrm{C} 3-\mathrm{N} 1-\mathrm{C} 1$ & $120.2(2)$ \\
\hline $\mathrm{C} 15-\mathrm{C} 10-\mathrm{C} 9$ & $121.9(2)$ & $\mathrm{C} 2-\mathrm{N} 1-\mathrm{C} 1$ & $118.6(2)$ \\
\hline $\mathrm{C} 12-\mathrm{C} 11-\mathrm{C} 10$ & $123.1(2)$ & $\mathrm{C} 9-\mathrm{N} 2-\mathrm{C} 6$ & $123.9(2)$ \\
\hline $\mathrm{C} 12-\mathrm{C} 11-\mathrm{H} 11$ & 118.5 & $\mathrm{C} 13-\mathrm{N} 3-\mathrm{C} 16$ & $121.5(3)$ \\
\hline $\mathrm{C} 10-\mathrm{C} 11-\mathrm{H} 11$ & 118.5 & $\mathrm{C} 13-\mathrm{N} 3-\mathrm{C} 18$ & $121.1(3)$ \\
\hline $\mathrm{C} 11-\mathrm{C} 12-\mathrm{C} 13$ & $120.3(2)$ & $\mathrm{C} 16-\mathrm{N} 3-\mathrm{C} 18$ & $117.4(2)$ \\
\hline $\mathrm{C} 11-\mathrm{C} 12-\mathrm{H} 12$ & 119.8 & $\mathrm{C} 15-\mathrm{O} 1-\mathrm{H} 1$ & 109.5 \\
\hline $\mathrm{C} 13-\mathrm{C} 12-\mathrm{H} 12$ & 119.8 & & \\
\hline $\mathrm{N} 1-\mathrm{C} 3-\mathrm{C} 4-\mathrm{C} 5$ & $178.6(3)$ & $\mathrm{C} 13-\mathrm{C} 14-\mathrm{C} 15-\mathrm{C} 10$ & $1.1(4)$ \\
\hline $\mathrm{C} 8-\mathrm{C} 3-\mathrm{C} 4-\mathrm{C} 5$ & $-0.3(4)$ & $\mathrm{C} 11-\mathrm{C} 10-\mathrm{C} 15-\mathrm{O} 1$ & $177.5(3)$ \\
\hline $\mathrm{C} 3-\mathrm{C} 4-\mathrm{C} 5-\mathrm{C} 6$ & $0.5(4)$ & $\mathrm{C} 9-\mathrm{C} 10-\mathrm{C} 15-\mathrm{O} 1$ & $-4.4(4)$ \\
\hline $\mathrm{C} 4-\mathrm{C} 5-\mathrm{C} 6-\mathrm{C} 7$ & $-0.2(4)$ & $\mathrm{C} 11-\mathrm{C} 10-\mathrm{C} 15-\mathrm{C} 14$ & $-2.3(4)$ \\
\hline $\mathrm{C} 4-\mathrm{C} 5-\mathrm{C} 6-\mathrm{N} 2$ & $-179.1(3)$ & $\mathrm{C} 9-\mathrm{C} 10-\mathrm{C} 15-\mathrm{C} 14$ & $175.8(3)$ \\
\hline $\mathrm{C} 5-\mathrm{C} 6-\mathrm{C} 7-\mathrm{C} 8$ & $-0.3(4)$ & $\mathrm{C} 8-\mathrm{C} 3-\mathrm{N} 1-\mathrm{C} 2$ & $-6.4(5)$ \\
\hline $\mathrm{N} 2-\mathrm{C} 6-\mathrm{C} 7-\mathrm{C} 8$ & $178.7(2)$ & $\mathrm{C} 4-\mathrm{C} 3-\mathrm{N} 1-\mathrm{C} 2$ & $174.8(3)$ \\
\hline $\mathrm{C} 6-\mathrm{C} 7-\mathrm{C} 8-\mathrm{C} 3$ & $0.5(4)$ & $\mathrm{C} 8-\mathrm{C} 3-\mathrm{N} 1-\mathrm{C} 1$ & $-179.2(3)$ \\
\hline $\mathrm{N} 1-\mathrm{C} 3-\mathrm{C} 8-\mathrm{C} 7$ & $-179.0(3)$ & $\mathrm{C} 4-\mathrm{C} 3-\mathrm{N} 1-\mathrm{C} 1$ & $2.1(4)$ \\
\hline $\mathrm{C} 4-\mathrm{C} 3-\mathrm{C} 8-\mathrm{C} 7$ & $-0.2(4)$ & $\mathrm{C} 10-\mathrm{C} 9-\mathrm{N} 2-\mathrm{C} 6$ & $-177.7(2)$ \\
\hline $\mathrm{N} 2-\mathrm{C} 9-\mathrm{C} 10-\mathrm{C} 11$ & $179.7(2)$ & $\mathrm{C} 7-\mathrm{C} 6-\mathrm{N} 2-\mathrm{C} 9$ & $170.0(2)$ \\
\hline $\mathrm{N} 2-\mathrm{C} 9-\mathrm{C} 10-\mathrm{C} 15$ & $1.7(4)$ & $\mathrm{C} 5-\mathrm{C} 6-\mathrm{N} 2-\mathrm{C} 9$ & $-11.0(4)$ \\
\hline
\end{tabular}




$\begin{array}{llll}\mathrm{C} 15-\mathrm{C} 10-\mathrm{C} 11-\mathrm{C} 12 & 1.4(4) & \mathrm{C} 14-\mathrm{C} 13-\mathrm{N} 3-\mathrm{C} 16 & -15.8(5) \\ \mathrm{C} 9-\mathrm{C} 10-\mathrm{C} 11-\mathrm{C} 12 & -176.7(3) & \mathrm{C} 12-\mathrm{C} 13-\mathrm{N} 3-\mathrm{C} 16 & 165.5(3) \\ \mathrm{C} 10-\mathrm{C} 11-\mathrm{C} 12-\mathrm{C} 13 & 0.8(4) & \mathrm{C} 14-\mathrm{C} 13-\mathrm{N} 3-\mathrm{C} 18 & 167.5(3) \\ \mathrm{C} 11-\mathrm{C} 12-\mathrm{C} 13-\mathrm{N} 3 & 176.7(3) & \mathrm{C} 12-\mathrm{C} 13-\mathrm{N} 3-\mathrm{C} 18 & -11.3(5) \\ \mathrm{C} 11-\mathrm{C} 12-\mathrm{C} 13-\mathrm{C} 14 & -2.1(4) & \mathrm{C} 17-\mathrm{C} 16-\mathrm{N} 3-\mathrm{C} 13 & 97.4(4) \\ \mathrm{N} 3-\mathrm{C} 13-\mathrm{C} 14-\mathrm{C} 15 & -177.6(3) & \mathrm{C} 17-\mathrm{C} 16-\mathrm{N} 3-\mathrm{C} 18 & -85.7(4) \\ \mathrm{C} 12-\mathrm{C} 13-\mathrm{C} 14-\mathrm{C} 15 & 1.2(4) & \mathrm{C} 19-\mathrm{C} 18-\mathrm{N} 3-\mathrm{C} 13 & 91.4(4) \\ \mathrm{C} 13-\mathrm{C} 14-\mathrm{C} 15-\mathrm{O} 1 & -178.8(3) & \mathrm{C} 19-\mathrm{C} 18-\mathrm{N} 3-\mathrm{C} 16 & -85.5(4)\end{array}$

Hydrogen-bond geometry $\left(A,{ }^{\circ}\right)$

$\mathrm{Cg} 1$ and $\mathrm{Cg} 2$ are the centroids of rings $\mathrm{C} 3-\mathrm{C} 8$ and $\mathrm{C} 10-\mathrm{C} 15$, respectively.

\begin{tabular}{lllll}
\hline$D-\mathrm{H} \cdots A$ & $D-\mathrm{H}$ & $\mathrm{H} \cdots A$ & $D \cdots A$ & $D-\mathrm{H} \cdots A$ \\
\hline $\mathrm{O} 1-\mathrm{H} 1 \cdots \mathrm{N} 2$ & 0.82 & 1.85 & $2.585(3)$ & 148 \\
$\mathrm{C} 11-\mathrm{H} 11 \cdots C g 1^{\mathrm{i}}$ & 0.93 & 2.71 & $3.517(3)$ & 145 \\
$\mathrm{C} 17-\mathrm{H} 17 B \cdots C g 2^{\mathrm{ii}}$ & 0.96 & 2.90 & $3.743(5)$ & 147 \\
\hline
\end{tabular}

Symmetry codes: (i) $-x, y+1 / 2,-z+1$; (ii) $-x, y-1 / 2,-z+2$. 